\title{
Microscopic origin of the anomalous Hall effect in noncollinear kagome magnets
}

\author{
Oliver Busch $₫$, Börge Göbel $\odot{ }^{*}$, and Ingrid Mertig \\ Institut für Physik, Martin-Luther-Universität Halle-Wittenberg, D-06099 Halle (Saale), Germany
}

(Received 28 May 2020; accepted 1 July 2020; published 21 July 2020)

\begin{abstract}
The anomalous Hall effect is commonly considered a signature of ferromagnetism. However, recently, an enormous anomalous Hall conductivity was measured in the compensated kagome magnets $\mathrm{Mn}_{3} \mathrm{Sn}$ and $\mathrm{Mn}_{3} \mathrm{Ge}$. The occurrence of this effect is allowed by the magnetic point group of these materials; however, its emergence is still lacking a microscopic explanation. Herein we show that the spin-orbit coupling and an out-of-plane tilting of the texture are equivalent for several kagome magnets. Consequently, a coplanar system with spin-orbit coupling behaves as if it were virtually noncoplanar. We show via tight-binding model calculations that the Hall effect can mainly be interpreted as a topological Hall effect generated by the opening angle of the virtually tilted texture. Furthermore, upon tilting the fixed texture out of the kagome plane, we find a critical tilting angle for which the Hall conductivity vanishes for all energies. In this case, the Hamiltonian is invariant under a combined time-reversal and mirror symmetry, because the virtual texture is coplanar.
\end{abstract}

DOI: 10.1103/PhysRevResearch.2.033112

\section{INTRODUCTION}

The Hall effect of electrons is one of the most intensely investigated effects in solid-state physics. It describes the transverse deflection of moving conduction electrons in a Hall bar when time-reversal symmetry is broken. Besides the conventional Hall effect which is caused by an externally applied magnetic field [1], the anomalous Hall effect [2] has become a signature of ferromagnets [3]. This effect can be caused by the skew-scattering [4,5] and side-jump [6] mechanisms. Both contributions are extrinsic; they are related to the spin-orbit coupling at defects [3]. Besides, even in a perfect sample, an anomalous Hall effect can arise intrinsically [7] due to the occurrence of a reciprocal-space Berry curvature [8,9]. Typically, all three contributions are related to a net magnetization; it breaks a set of time-reversal and spatial symmetries so that a reciprocal-space Berry curvature can arise. Furthermore, a Berry curvature can also be caused by noncollinear magnetic textures with a net scalar spin chirality [10], i.e., certain noncoplanar textures like magnetic skyrmions [11,12]. The canted magnetic moments effectively generate an emergent magnetic field (or real-space Berry curvature) that causes the so-called topological Hall effect [13].

Over the last years, a straightforward microscopic understanding has been established for all three of the above presented contributions to the Hall effect. However, recently it was found that also materials without a net magnetization can exhibit an anomalous Hall effect [14-18], whose microscopic origin must be different from the anomalous Hall effect mentioned above. In several layered kagome materials a coplanar

\footnotetext{
*Corresponding author: boerge.goebel@physik.uni-halle.de
}

Published by the American Physical Society under the terms of the Creative Commons Attribution 4.0 International license. Further distribution of this work must maintain attribution to the author(s) and the published article's title, journal citation, and DOI. spin texture is observed with a vanishing net magnetization, and still tight-binding models and first-principle calculations predict finite anomalous Hall conductivities in agreement with magnetic point group analyses $[18,19]$. Recent experiments in $\mathrm{Mn}_{3} \mathrm{Sn}$ [16] and $\mathrm{Mn}_{3} \mathrm{Ge}$ [17], both members of the space group $P 6_{3} / m m c$ (No. 194) $[16,18]$, have reported large anomalous Hall conductivities of up to $500 \Omega^{-1} \mathrm{~cm}^{-1}$ confirming these predictions [20]. However, a straightforward microscopic understanding is still missing.

Herein we establish a microscopic explanation for the occurrence of a finite anomalous Hall effect in several kagome magnets. We show that the spin-orbit coupling for these systems is equivalent to a tilting of the magnetic moments out of the kagome plane. The Hall effect can then be explained by conventional means upon considering the net moment and the net scalar spin chirality of the virtually tilted texture instead of the actual texture. Using tight-binding calculations, we show that the topological Hall contribution (caused by the net scalar spin chirality) is dominant over the anomalous Hall contribution (caused by the net magnetic moment of the virtual texture). Furthermore, we show that the virtual tilting due to the spin-orbit coupling can be compensated by tilting the actual texture along the opposite out-of-plane direction. For a critical angle the virtual texture is coplanar, restoring the combined time-reversal and mirror symmetry of the Hamiltonian that forbids finite Hall conductivities.

\section{MODEL AND METHODS}

Throughout our study, we consider the tight-binding Hamiltonian as used in Ref. [14] in which the anomalous Hall effect in these kagome magnets has first been predicted,

$$
\begin{aligned}
H= & t \sum_{\langle i, j\rangle} c_{i}^{\dagger} c_{j}+m \sum_{i} c_{i}^{\dagger}\left(\boldsymbol{m}_{i} \cdot \boldsymbol{\sigma}\right) c_{i} \\
& +i \lambda \sum_{\langle i, j\rangle} c_{i}^{\dagger}\left(\boldsymbol{n}_{i j} \cdot \boldsymbol{\sigma}\right) c_{j} .
\end{aligned}
$$


(a)
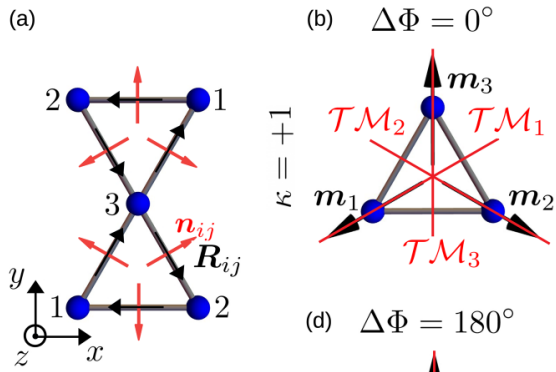

(d) $\Delta \Phi=180^{\circ}$

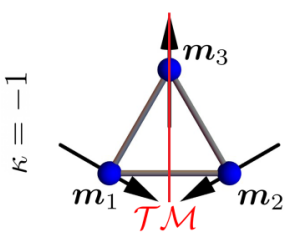

(c) $\Delta \Phi=90^{\circ}$

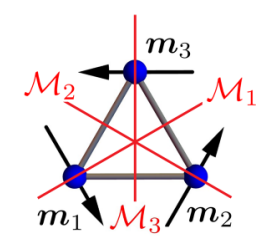

(e)

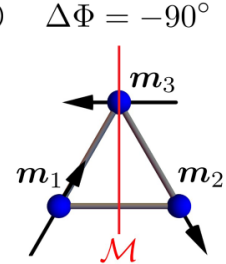

FIG. 1. Coplanar magnetic textures on a kagome lattice. (a) The three basis atoms have four nearest neighbors each. For each of these bonds the spin-orbit vector $\boldsymbol{n}_{i j}=-\boldsymbol{n}_{j i}$ is indicated (red; the black arrow indicates the direction $i j$ versus $j i$ ). (b) The three basis atoms and magnetic moments $\boldsymbol{m}_{i}$ (indicated by arrows) in the coplanar radial configuration. The unit cell has three symmetry planes indicating a combined time-reversal and mirror symmetry (red). The texture is characterized by a positive vector spin chirality $\kappa=+1$ and an in-plane offset $\Delta \Phi=0^{\circ}$, as indicated. (c) The toroidal phase. All moments are locally rotated by $90^{\circ}$ compared to (b) giving $\Delta \Phi=$ $90^{\circ}$. This magnetic texture has three mirror symmetry planes. (d), (e) Textures with a negative vector chirality are shown. They only have one symmetry plane each.

The first term represents the hopping of an electron from lattice site $j$ to $i$ with the amplitude $t$. The second term describes the Hund's coupling of electron spin and magnetic texture $\left\{\boldsymbol{m}_{i}\right\}$. The strength of this interaction is $m=1.7 t$ throughout this paper. The third term represents the spin-orbit coupling, as introduced in Ref. [14], similar to the spin-orbit coupling in graphene [21]. The term preserves the inversion symmetry, but accounts for the difference between left- and right-hand environments for electrons hopping along different paths in a kagome layer [14]. The vectors $\boldsymbol{n}_{i j}=-\boldsymbol{n}_{j i}$ are visualized in Fig. 1(a) (red arrows).

This minimal model comprises a subset of the full Hamiltonian. Only a few relevant bands near the Fermi energy are considered and the magnetic texture $\left\{\boldsymbol{m}_{i}\right\}$ is assumed to be constituted by energetically lower states and is fixed. Still, the model allows us to establish an understanding for the emerging Hall effect since it has the same symmetry as the complete system.

Diagonalizing this Hamiltonian gives the band structure as eigenvalues $E_{n}(\boldsymbol{k})$ and the eigenvectors $\left|n_{\boldsymbol{k}}\right\rangle \equiv\left|u_{n}(\boldsymbol{k})\right\rangle$ from which the intrinsic contribution to the Hall conductivity can be calculated [3],

$$
\sigma_{x y}\left(E_{F}\right)=-\frac{e^{2}}{h} \frac{1}{2 \pi} \sum_{n} \int_{E(\boldsymbol{k}) \leqslant E_{F}} \Omega_{n}^{(z)}(\boldsymbol{k}) d^{2} k .
$$

We treat the Fermi energy $E_{F}$ as a parameter that can be tuned by doping or application of a gate voltage. $\Omega_{n}^{(z)}(\boldsymbol{k})$ is the $z$

component of the reciprocal-space Berry curvature of band $n$,

$$
\Omega_{n}^{(z)}(\boldsymbol{k})=-2 \operatorname{Im} \sum_{m \neq n} \frac{\left\langle n_{\boldsymbol{k}}\left|\partial_{k_{x}} H_{\boldsymbol{k}}\right| m_{\boldsymbol{k}}\right\rangle\left\langle m_{\boldsymbol{k}}\left|\partial_{k_{y}} H_{\boldsymbol{k}}\right| n_{\boldsymbol{k}}\right\rangle}{\left[E_{n}(\boldsymbol{k})-E_{m}(\boldsymbol{k})\right]^{2}} .
$$

Next, we introduce the different configurations of the considered magnetic texture. The unit cell of a kagome layer consists of three atoms. The three magnetic moments have an angle of $120^{\circ}$ with respect to each other. The polar angle of their orientation $\Phi_{i}$ and the polar angle of their position vector $\phi_{i}$ (center of the coordinate system in the center of the plaquette) are related by

$$
\Phi_{i}=\kappa \phi_{i}+\Delta \Phi
$$

for all basis atoms $i=1,2,3$. Here $\kappa= \pm 1$ is the vector spin chirality. We discuss the following experimentally observed kagome magnets: For a positive vector spin chirality $\kappa=+1$ there exist two different configurations of $\mathrm{Mn}_{3} Z \mathrm{~N}(Z=\mathrm{Ni}$, Ga, Zn, Sn, Ag, Rh, Pt) [22,23] or $\mathrm{Mn}_{3} X$ ( $X=\mathrm{Rh}, \mathrm{Ir}, \mathrm{Pt}$ ) [24] [radial order $\Delta \Phi=0^{\circ}, 180^{\circ}$ and toroidal order $\Delta \Phi= \pm 90^{\circ}$ shown in Figs. 1(b) and 1(c)], and for a negative vector spin chirality $\kappa=-1$ there exist two different configuration of $\mathrm{Mn}_{3} Y(Y=\mathrm{Sn}, \mathrm{Ge}, \mathrm{Ga})[25]\left[\Delta \Phi=0^{\circ}, 180^{\circ}\right.$ and $\Delta \Phi=$ $\pm 90^{\circ}$ shown in Figs. 1(d) and 1(e)]. Additionally, the tightbinding model allows us to arbitrarily vary the in-plane offset angle $\Delta \Phi$, similarly to the study in Ref. [26], as well as the azimuthal angle $\theta$ [cf. Fig. 3(a); $\theta=90^{\circ}$ for coplanar textures] like in Ref. [14] in order to analyze the canted magnetic textures later in this paper.

Before we present our results, we want to stress that we consider three fundamentally different types of textures throughout our paper: the fixed magnetic texture, the electron spin texture, and a virtual texture. The fixed magnetic texture $\left\{\boldsymbol{m}_{i}\right\}$ is a real-space texture that is formed by localized magnetic moments. This texture is predefined and directly enters the Hamiltonian (1). It is defined by the in-plane orientation given in Eq. (3) and the azimuthal angle $\theta$. Besides, the itinerant spin magnetic moments of the conduction electrons form a texture in reciprocal space. We obtain this so called spin texture by calculating the spin expectation value

$$
\left\langle\boldsymbol{s}_{n}(\boldsymbol{k})\right\rangle=\left\langle n_{\boldsymbol{k}}|\boldsymbol{\Sigma}| n_{k}\right\rangle,
$$

where $\boldsymbol{\Sigma}=\operatorname{diag}(\sigma, \ldots, \sigma)$ is given by the vector of Pauli matrices $\sigma$. For a deeper understanding we can also analyze the spin texture that originates from a single atom. In this case we use $\boldsymbol{\Sigma}=\operatorname{diag}(\mathbf{0}, \ldots, \boldsymbol{\sigma}, \ldots, \boldsymbol{0})$. As we will present later in this paper, the transport properties are only loosely related to the fixed magnetic texture or the spin texture of electrons. Instead, the spin-orbit coupling gives rise to a virtual texture $\left\{\tilde{\boldsymbol{m}}_{i}\right\}$ which is effectively tilted with respect to the fixed texture out of the kagome plane. This virtual texture is not measurable but is determined by the symmetry of the system. Therefore, it determines the emerging Hall effects and can be utilized to explain whether and how certain symmetries are broken.

\section{RESULTS AND DISCUSSION}

In the following we present calculations of the band structure and the anomalous Hall conductivity within the 

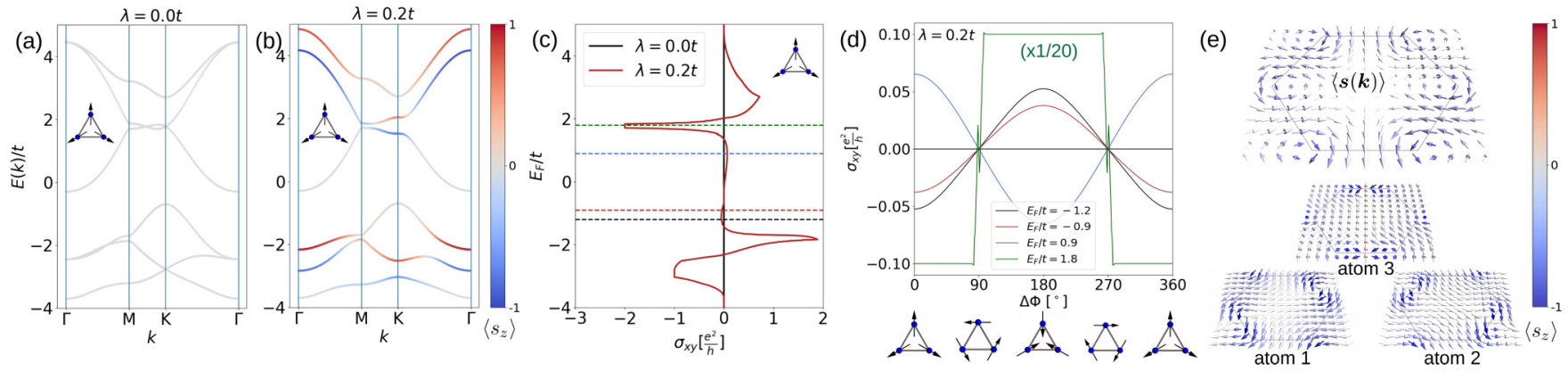

FIG. 2. Band structure and anomalous Hall conductivity for the coplanar configurations, $\theta=90^{\circ}$. (a) The band structure of the radial configuration, $\Delta \Phi=0$, when spin-orbit interaction is not taken into account. The out-of-plane spin expectation value $\left\langle s_{z}\right\rangle$ of all bands and $k$ points vanishes (gray). (b) Similar to (a) but the spin-orbit interaction is now taken into account. Degeneracies are lifted and the spin expectation value now has a finite out-of-plane component (red, positive; blue, negative). (c) The anomalous Hall conductivity corresponding to the band structure in (b). (d) shows the anomalous Hall conductivity for various Fermi energies [colored lines indicated in (c)] upon locally rotating each moment around the local $z$ axis. Cartoons of the texture for several angles $\Delta \Phi$ are shown below. (e) The spin expectation value for the energetically lowest band in (b). The color encodes the out-of-plane component. Below, the atom-resolved spin expectation value is shown.

framework of the presented tight-binding model. We focus here on the $\kappa=+1$ phase as for example $\mathrm{Mn}_{3} \mathrm{Pt}$. Later we will briefly refer to the $\kappa=-1$ phase as well, which shows similar results and for which the same arguments hold that we will establish in the following.

For the $\kappa=+1$ phase, we begin by discussing different coplanar configurations and with a review of the existing literature. To reveal the mechanism for the emergence of this effect-the main subject of our paper-we analyze the expectation value of the conduction electrons' spin, establish the equivalence of the spin-orbit coupling and an out-of-plane tilting of the moments, and even consider fixed magnetic textures that have been tilted out of the kagome plane.

\section{A. Band structure and anomalous Hall conductivity of the coplanar radial configuration}

Before we consider magnetic systems, we start with a brief analysis of the structural kagome lattice. The three atoms in the unit cell of a nonmagnetic kagome layer without spinorbit coupling give rise to three spin-degenerate bands (band structure shown in Fig. 1 of the Supplemental Material [27]). One of these bands is ideally flat and the other two bands exhibit a band structure similar to graphene: They touch linearly at the Brillouin zone's $K$ points exhibiting Dirac cones. Since a magnetic texture is not considered yet, time-reversal symmetry is conserved and an anomalous Hall conductivity is absent.

Next, we will review the results for coplanar configurations, similar to Refs. [14,18,26,28]. When we consider the magnetic texture like in the radial phase of $\mathrm{Mn}_{3} \mathrm{Pt}$, as shown in Fig. 1(b), the spin degeneracy of the bands is lifted due to the broken time-reversal symmetry and six individual bands are visible [Fig. 2(a)]. Reminiscent of the Dirac cones of the original band structure, bands touch linearly at the $K$ points. Also, degeneracies are present at $\Gamma$. Here, in contrast to the Dirac points, the band structure is quadratic for all bands.

The mirror symmetry $M$ is broken in the system which is essential for the emergence of a Hall conductivity. However, since spin-orbit coupling is still not taken into account, the system has a combined time-reversal and mirror symmetry
$T M$ forbidding the occurrence of an anomalous Hall conductivity, since $\Omega_{n}^{(z)}(-\boldsymbol{k})=-\Omega_{n}^{(z)}(\boldsymbol{k})$ [14] [cf. red lines representing $T M$ symmetry planes in Fig. 1(b)]. The conduction electrons' spins partially align with the spatially dependent fixed magnetic texture at each lattice site (see Fig. 2(a) of the Supplemental Material [27]). Both spin texture and fixed magnetic texture are oriented in the kagome plane.

When spin-orbit interaction is considered, TM symmetry is broken (we establish how this symmetry is broken microscopically later in this paper) and the degeneracies in the band structure are lifted [red in Fig. 2(b)]. Away from the highsymmetry points, the band structure stays mostly unchanged. The breaking of TM symmetry leads to the emergence of an anomalous Hall conductivity [red in Fig. 2(c)] that exhibits a pronounced energy dependence. In the global band gaps the conductivity is quantized in units of $e^{2} / h$; i.e., a quantum anomalous Hall effect occurs [29]. The system becomes a Chern insulator.

\section{B. In-plane rotation of the magnetic texture}

A noncollinear coplanar magnetic texture does not always lead to the emergence of an anomalous Hall effect. When the magnetic moments $\left\{\boldsymbol{m}_{i}\right\}$ are collectively rotated around the local $z$ axis by $\Delta \Phi=90^{\circ}$, the alternative phase of materials like $\mathrm{Mn}_{3} \mathrm{Pt}$ is established. This system has three mirror symmetry planes [red in Fig. 1(c)] which protect the Dirac points and render the anomalous Hall effect zero, even upon considering spin-orbit coupling.

In Ref. [26] Zhou et al. have investigated the anomalous Hall conductivity for phases between these two states. We find similar results and conclude the following: For Fermi energies that are close to the band edges [red and blue curves in Fig. 2(d)], the signal is proportional to $\cos (\Delta \Phi)$, where $\Delta \Phi$ is the in-plane rotation angle $\left(\Delta \Phi=0^{\circ}, 180^{\circ}\right.$ corresponds to the radial phase and $\Delta \Phi= \pm 90^{\circ}$ corresponds to the toroidal phase). This observation is reasonable, since the projection of each moment on the potential mirror plane breaks the mirror symmetry; it is given by $\cos (\Delta \Phi)$ as well. Loosely speaking, the broken mirror symmetry does not only allow for the anomalous Hall effect to arise, but the "degree by which 
(a)

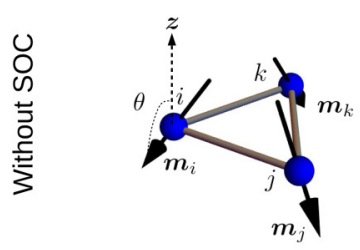

(f)

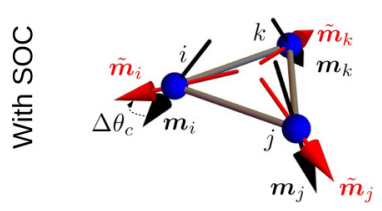

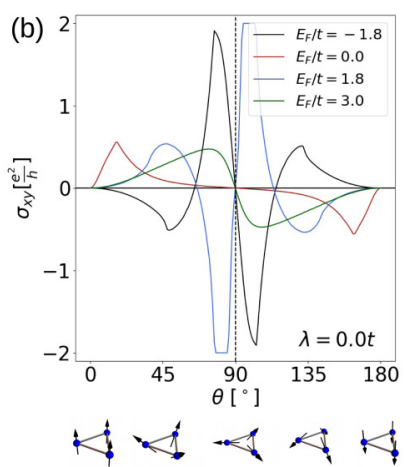
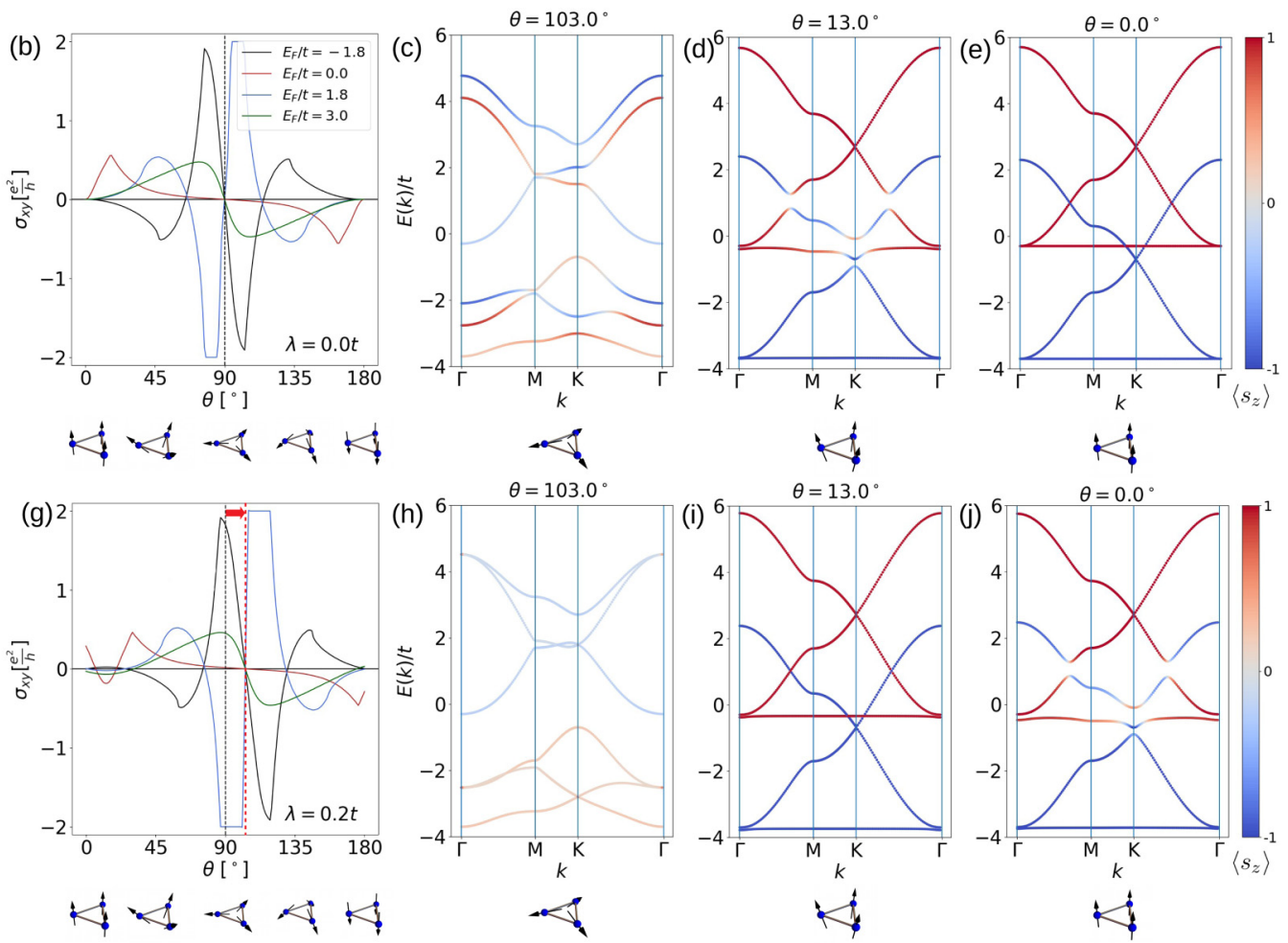

FIG. 3. Anomalous Hall conductivity under out-of-plane tilting of the fixed magnetic texture. In (a)-(e) spin-orbit coupling (SOC) has not been considered, whereas in (f)-(j) it is taken into account. (a) A noncollinear and noncoplanar configuration. Black arrows indicate the magnetic moments $\boldsymbol{m}_{i}$. (b) Hall conductivity versus azimuthal angle $\theta$. All four curves (different colors correspond to different Fermi energies) are antisymmetric with respect to $\theta=90^{\circ}$ which is the coplanar configuration as indicated by the cartoons below. (c)-(e) Band structures with out-of-plane components of the spin expectation value (red, positive; blue, negative) for different azimuthal angles $\theta$ as indicated. (f) The texture from (a) in black. Additionally, the virtual magnetic texture $\left\{\tilde{\boldsymbol{m}}_{i}\right\}$ is shown in red. This texture arises due to the spin-orbit coupling, as explained in the main text, and it is characterized by a reduced azimuthal angle $\theta-\Delta \theta_{c}$. (g) Same as (b), but spin-orbit interaction is taken into account. Here the curves are antisymmetric with respect to $\theta_{c}=90^{\circ}+\Delta \theta_{c} \approx 103^{\circ}$. (h)-(j) Same as (c)-(e), but spin-orbit interaction was considered as in panel $(\mathrm{g})$.

the symmetry is broken" even determines the magnitude of the effect. Furthermore, this projection also determines the size of the band gaps due to spin-orbit coupling. For the toroidal phase $\left(\Delta \Phi= \pm 90^{\circ}\right)$, the band structure is unaffected by the spin-orbit coupling (band structures are shown in Fig. 3 of the Supplemental Material [27]).

The $\cos (\Delta \Phi)$ behavior does not hold for Fermi energies close to the band edge, let alone in the band gap: the black curve in Fig. 2(d) is not perfectly cosinusoidal and the green curve at $E_{F}=1.8 t$ is a step function, since the corresponding Fermi energy is located in a band gap (an opened Dirac point for $\Delta \Phi \neq \pm 90^{\circ}$ ) and the Hall conductivity must be quantized.

Summarizing up to this point, we have presented how the anomalous Hall conductivity for noncollinear coplanar magnetic textures can be related to the breaking of $M$ and $T M$ symmetries, which is where the limit of the existing literature is reached. In the following, we will unravel the underlying microscopic mechanism.

\section{Out-of-plane spin tilting of the conduction electrons}

First, we consider a quantity that allows us to explain the emergence of the anomalous Hall effect in coplanar systems microscopically: a tilted spin texture formed by the conduction electrons.

As mentioned, without spin-orbit interaction, the conduction electrons' spins remain in the plane, partially aligned with the fixed magnetic texture [cf. completely gray bands in Fig. 2(a)]. When the spin-orbit interaction is taken into account in the toroidal phase, this is unchanged and the Hall conductivity stays zero (cf. Fig. 3 of the Supplemental Material [27]). However, in the radial phase, the spins start to cant out of the plane [red and blue in Fig. 2(b)]. This canting is $k$ and band dependent, and general trends can be observed: For the first (energetically lowest) and fourth band, the tilting is along the $-z$ direction, while for the third and sixth (energetically highest) band it is along the $+z$ direction. This relates quite well to the anomalous Hall signal shown in Fig. 2(c): Within the first and fourth band, the conductivity is negative (or close to zero if positive) and within the third and sixth band it is positive. For energies within the second or fifth band, where the out-of-plane spin component is positive and negative, the anomalous conductivity changes sign.

Even though we have not yet explained what causes the tilting, one can register that the spin moment of the conduction electrons is nonzero in this model, even though the fixed magnetic texture is coplanar. For this reason, one may argue 
that it is clear that a finite Hall conductivity is calculated by conventional means: the total texture has a net magnetization and even a net scalar spin chirality $\chi_{s}=s_{i} \cdot\left(s_{j} \times s_{k}\right)$ which give rise to an anomalous and topological Hall effect, respectively.

While this explanation seems reasonable a first glance, in the following, we vary the azimuthal angle of the fixed magnetic texture and find that there exist cases for which this explanation does not hold: Even for a combined texture with a net moment and a net scalar spin chirality the Hall effect can be absent and for a completely compensated system the Hall effect can be finite. Therefore, next we analyze the system on the Hamiltonian level to unravel the microscopic mechanism and to show how the TM symmetry is broken precisely and how it can even be restored for certain noncoplanar magnetic textures.

\section{Out-of-plane rotation of the magnetic texture}

In the following, we tilt the fixed texture out of the kagome plane (azimuthal angle $\theta$ ) which, in the end, allows us to establish the actual microscopic explanation for the emergence of the anomalous Hall effect in kagome magnets: the existence of a virtual texture $\left\{\tilde{\boldsymbol{m}}_{i}\right\}$ that is tilted with respect to the actual magnetic texture $\left\{\boldsymbol{m}_{i}\right\}$ due to the spin-orbit coupling.

First we present and discuss the results upon changing $\theta$ without taking spin-orbit interaction into account [Fig. 3(b)]. This reference system exhibits a pure topological Hall effect since the anomalous Hall effect is absent by definition. For $\theta=0^{\circ}$ and $180^{\circ}$, the localized moments are parallel and point out of the kagome plane. For $\theta=90^{\circ}$ the moments are coplanar as described above. In both cases the scalar spin chirality vanishes and a topological Hall effect does not emerge. For all other angles the scalar spin chirality

$$
\chi_{s}=\boldsymbol{m}_{i} \cdot\left(\boldsymbol{m}_{j} \times \boldsymbol{m}_{k}\right)=\frac{3 \sqrt{3}}{2} \cos \theta \sin ^{2} \theta
$$

( $i, j$, and $k$ are the lattice sites of a kagome plaquette) is finite and so is the calculated Hall conductivity (except for single angles where contributions from different bands compensate). For all energies, the angular-dependent curves are antisymmetric with respect to $\theta=90^{\circ}$, due to the antisymmetry of the scalar spin chirality. While continuously changing the azimuthal angle $\theta$, the band structure changes considerably. For this reason, a fixed energy may even shift through band gaps into other bands as is seen in the blue curve for $E_{F}=$ $1.8 t$. When the Fermi energy is located in the gap, the Hall conductivity is quantized. We repeated these calculations also for fixed occupation numbers (see Fig. 4 of the Supplemental Material [27]). The curves remained antisymmetric.

When we consider the spin-orbit interaction [cf. Fig. 3(g)], the curves change and the configurations $\theta=0^{\circ}, 90^{\circ}$, and $180^{\circ}$ now yield a finite signal in agreement with the above presented findings that the radial type of the coplanar configuration $\left(\theta=90^{\circ}\right)$ exhibits an anomalous Hall effect and compatible with the typical observation that a collinear ferromagnet $\left(\theta=0^{\circ}, 180^{\circ}\right)$ exhibits an anomalous Hall effect when spin-orbit interaction is present. Generally, the shape of this angular-dependent curve looks similar to the case without spin-orbit coupling. However, besides minor changes near the ferromagnetic configurations, it is mainly shifted. The curve is still antisymmetric, not with respect to $\theta=90^{\circ}$, but shifted by about $\Delta \theta_{c}=13^{\circ}$ to an azimuthal angle $\theta_{c}=103^{\circ}$. This critical angle is parameter dependent, and we will precisely derive it later in this paper.

The systems for the two angles $\theta=13^{\circ}$ and $\theta=103^{\circ}$ are special in several regards: the Hall conductivity vanishes, the Dirac points are reestablished, and the reciprocal-space spin texture is homogeneous, (anti)parallel to the fixed texture [homogeneous light colors in Fig. 3(h) and homogeneous bright colors in Fig. 3(i)]. For all other configurations, even for the coplanar texture $\left(\theta=90^{\circ}\right)$ or the ferromagnetic configuration $\left(\theta=0^{\circ}, 180^{\circ}\right)[$ Fig. 3(j)], none of these three features are fulfilled.

\section{E. Microscopic mechanism: Tilted virtual texture hidden in the Hamiltonian}

These findings point toward a higher symmetry for the two special configurations characterized by $\theta=\Delta \theta_{c}$ and $\theta=$ $\theta_{c}=\Delta \theta_{c}+90^{\circ}$, even though the texture itself does not have a higher symmetry than the other textures (they have an even lower symmetry compared to the coplanar or ferromagnetic configuration).

As we show, this stems from the fact that the electronic properties of the system are not determined by the fixed texture, the conduction electrons' spin texture, or a combined texture, but by a virtual texture characterized by the Hamiltonian. This virtual texture $\left\{\tilde{\boldsymbol{m}}_{i}\right\}$ arises, since the spin-orbit coupling in this system is equivalent to a canting of the fixed magnetic texture $\left\{\boldsymbol{m}_{i}\right\}$ out of the kagome plane [cf. red and black arrows in Figs. 3(a) and 3(f)], as we summarize in the following. The transformation is shown in the Appendix in detail.

The full Hamiltonian (1) used throughout this paper can be transformed to

$$
\begin{aligned}
H= & \sum_{\langle i, j\rangle} t_{i j}^{\mathrm{eff}} d_{i}^{\dagger} d_{j}+m \sum_{i} d_{i}^{\dagger}\left(\tilde{\boldsymbol{m}}_{i} \cdot \boldsymbol{\sigma}\right) d_{i} \\
& +i \tilde{\lambda} \sum_{\langle i, j\rangle} d_{i}^{\dagger}\left(\boldsymbol{n}_{i j} \cdot \boldsymbol{\sigma}\right) d_{j},
\end{aligned}
$$

by applying a unitary transformation $U_{i}$ (new electron operators are $d_{i}$ ), that rotates the coordinate system at each lattice site. The new $z$ axis of the coordinate system at lattice site $i$ has an angle $\alpha$ with respect to the initial $z$ axis and has been tilted along the direction of the magnetic moment $\boldsymbol{m}_{i}$. A sketch of this rotation is shown in Fig. 6 as part of the Appendix.

This transformation, of course, leaves the physical properties unchanged but the spin and the magnetic moments are measured differently. In this lattice-site dependent coordinate system, the azimuthal angle of the fixed magnetic texture is effectively decreased by $\alpha$,

$$
\tilde{\boldsymbol{m}}_{i}=\left(\begin{array}{c}
\cos \left(\Phi_{i}\right) \sin (\theta-\alpha) \\
\sin \left(\Phi_{i}\right) \sin (\theta-\alpha) \\
\cos (\theta-\alpha)
\end{array}\right) .
$$

When the transformation is applied to the hopping term and the spin-orbit coupling, the (now spin-dependent) hopping amplitude is modified as given in the Appendix but most 

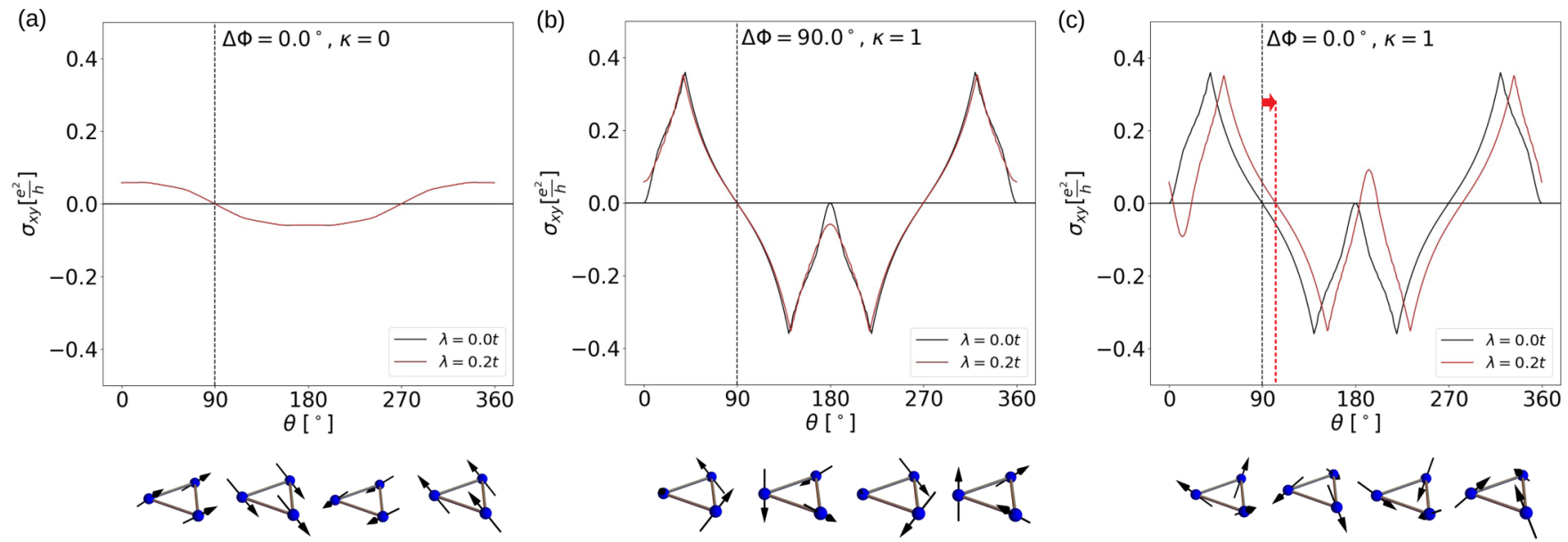

FIG. 4. Different contributions to the Hall effect for different magnetic textures. (a) The Hall conductivity for a collinear magnetic texture is shown under variation of the azimuthal angle $\theta$. Only a conventional anomalous Hall effect proportional to $m_{z}$ of the fixed magnetic texture is present when spin-orbit interaction is considered. (b) The toroidal texture is considered. Upon variation of $\theta$ the scalar spin chirality $\chi_{s}$ is finite and an additional topological Hall effect arises that is much larger than the anomalous Hall effect. (c) The radial texture is considered, as discussed above. The curve from the toroidal texture is mainly shifted by $\Delta \theta_{c}=13^{\circ}$ which characterizes the nature of the "new" anomalous Hall effect in this material class. All calculations were performed at a constant occupation number of $n_{\text {occ }}=3.4$.

importantly both terms now contribute with off-diagonal elements that can be identified as an effective spin-orbit coupling. For the radial phase it is quantified by

$$
\tilde{\lambda}=-\frac{\sqrt{3}}{2} \sin (\alpha) t+\cos (\alpha) \lambda .
$$

This transformation shows that the spin-orbit interaction and a texture that is tilted out of the kagome plane have the same effect in this system. Especially, a critical angle $\alpha_{c}$ exists, for which the effective spin-orbit coupling is compensated, $\tilde{\lambda}=0$. This angle determines the effective tilting angle $-\Delta \theta_{c}$ of the virtual texture $\left\{\tilde{\boldsymbol{m}}_{i}\right\}$ compared to the fixed texture $\left\{\boldsymbol{m}_{i}\right\}$ [cf. Fig. 3(f)] that arises due to spin-orbit coupling. For the phase with positive vector spin chirality, $\kappa=+1$, the critical compensation angle of the magnetic texture for which the virtually tilted texture is coplanar is

$$
\Delta \theta_{c}=\alpha_{c}=\arctan \left[\frac{\lambda}{t \sqrt{3} / 2} \cos (\Delta \Phi)\right] ;
$$

see the Appendix. The $\lambda$ and $\Delta \Phi$ dependencies have been confirmed numerically in Fig. 5 of the Supplemental Material [27]. The numerically determined data points have been determined by looking at the avoided crossings that form Dirac points at the critical angle. Furthermore, the Hall conductivity vanishes for all energies at these angles.

In summary, we have shown that the spin-orbit coupling term can be totally compensated by tilting the texture by $\Delta \theta_{c}$ along the azimuthal direction (and also modifying the hopping). This means that the consequences of the spin-orbit coupling and a tilted texture are equivalent in this system: A fixed texture $\left\{\boldsymbol{m}_{i}\right\}$ that is coplanar, $\theta=90^{\circ}$, under the influence of spin-orbit coupling, $\lambda \neq 0$, behaves like a tilted texture $\left\{\tilde{\boldsymbol{m}}_{i}\right\}$ in a system without spin-orbit coupling, $\tilde{\lambda}=0$, thereby explaining the emergence of the anomalous Hall effect in the coplanar system. Likewise, a texture $\left\{\boldsymbol{m}_{i}\right\}$ with a critical azimuthal angle $\theta_{c}=90^{\circ}+\Delta \theta_{c}$ with spin-orbit coupling, $\lambda \neq 0$, behaves like a texture $\left\{\tilde{\boldsymbol{m}}_{i}\right\}$ that is coplanar, $\theta=90^{\circ}$, in a system without spin-orbit coupling, $\tilde{\lambda}=0$, explaining why the Hall conductivity vanishes for $\theta=103^{\circ}$ in Fig. 3(g). In a similar way, this explains why the configuration characterized by $\theta=13^{\circ}$ in Fig. 3(i) behaves like a ferromagnetic configuration, while the actual ferromagnetic configuration does not: The azimuthal angle of the virtual textures is reduced by $13^{\circ}$.

The two special configurations for $\theta=\Delta \theta_{c}=13^{\circ}$ and $\theta=\theta_{c}=\Delta \theta_{c}+90^{\circ}=103^{\circ}$ restore the $T M$ symmetry of the Hamiltonian that we initially discussed in the coplanar system without spin-orbit coupling. This symmetry leads to the closing of Dirac points and to the absence of the Hall conductivity for these particular configurations since it renders the reciprocal-space Berry curvature antisymmetric $\Omega_{n}^{(z)}(-\boldsymbol{k})=$ $-\Omega_{n}^{(z)}(\boldsymbol{k})$. On the other hand, for a coplanar fixed magnetic texture $\left(\theta=90^{\circ}\right)$ the virtual texture is tilted by $-\Delta \theta_{c}$. This virtual texture has a net magnetization and a scalar spin chirality and therefore gives rise to anomalous and topological Hall effects which are allowed by the breaking of the TM symmetry.

\section{F. Effective topological Hall effect}

Next, we want to show which mechanism of the virtually tilted texture is more relevant: a virtual anomalous Hall effect caused by the net moment of the virtual texture or a virtual topological Hall effect caused by the scalar spin chirality of the virtual texture.

To differentiate the calculated signal into anomalous and topological Hall effects, in the following, we compare the $\theta$ dependence of the Hall conductivity of the radial-type magnetic texture with the toroidal phase and a collinear phase, as presented in Fig. 4. Without spin-orbit coupling, the toroidal spin texture behaves equally to the radial-type texture, since both exhibit a purely topological Hall effect, that is proportional to the scalar spin chirality, which is independent of $\Delta \Phi$ [given in Eq. (5)]. The collinear configuration does not 

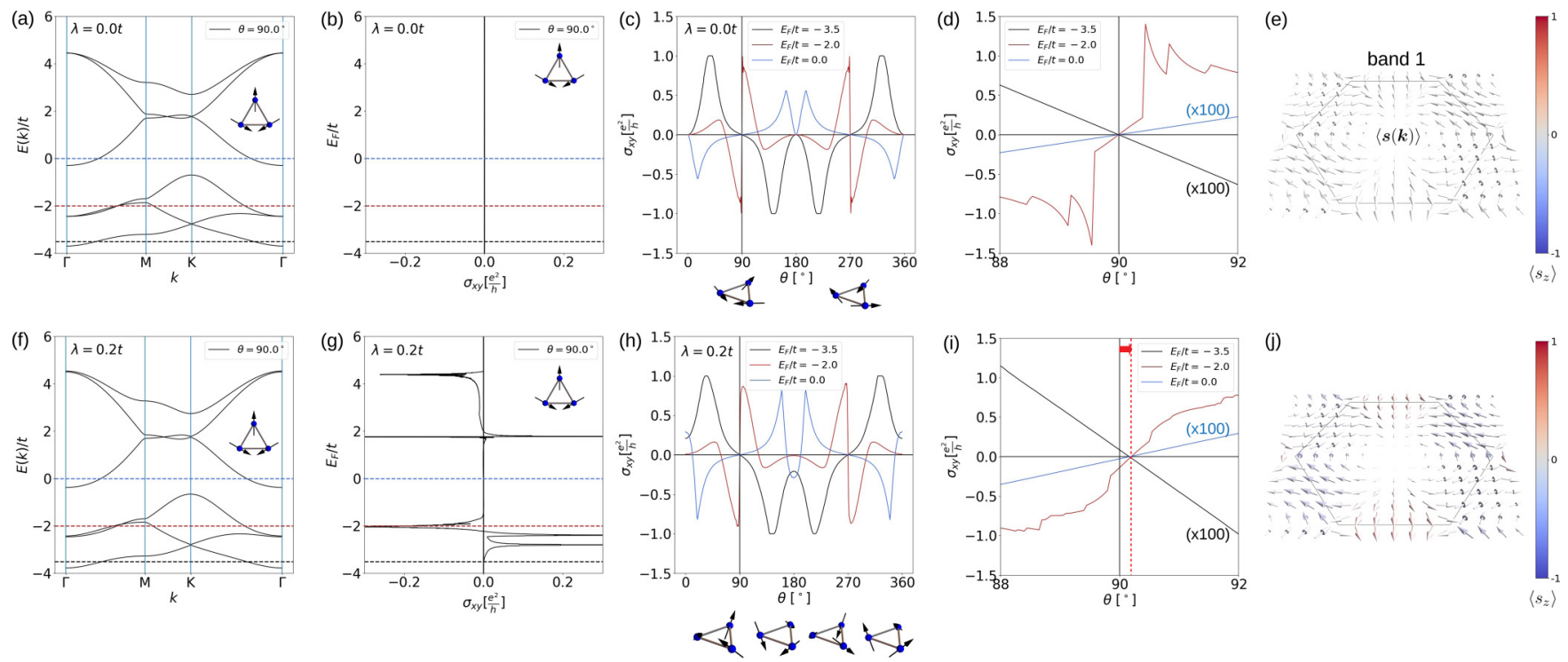

FIG. 5. Results for the phase of negative vector spin chirality, $\kappa=-1$ and $\Delta \Phi=180^{\circ}$. This is the texture that has been experimentally investigated in $\mathrm{Mn}_{3} \mathrm{Ge}$ [17]. (a)-(e) show the results without spin-orbit coupling. (a) The band structure, (b) the energy-resolved conductivity for the coplanar $\left(\theta=90^{\circ}\right)$ texture, (c) the Hall conductivity for different constant Fermi energies [colors as indicated in (a), (b)] in dependence on the azimuthal angle $\theta$. (d) shows a magnification of (c) near $\theta=90^{\circ}$. (e) The spin texture of the energetically lowest band for $\theta=90^{\circ}$. (f)-(j) show the same quantities when the spin-orbit interaction $\lambda=0.2 t$ is taken into account. Similarly to the result of the radial phase (as extensively presented in the paper), the virtual texture $\left\{\tilde{\boldsymbol{m}}_{i}\right\}$, determined by the Hamiltonian, is tilted due to the spin-orbit coupling. This also affects the reciprocal-space spin texture shown in (j).

exhibit a finite signal since the scalar spin chirality is always zero.

When spin-orbit coupling is taken into account, the anomalous Hall effect is also present. Conventionally, it is proportional to the out-of-plane magnetization $\cos \theta$ which is observable for the collinear configuration in panel (a), that exhibits a pure anomalous Hall effect. For the toroidal texture (b) the signal is symmetric with respect to $\theta=90^{\circ}$. The topological Hall effect and the (considerably smaller) anomalous Hall effect are present. In panel (c) the radial configuration is shown as discussed above. In addition to these two effects, a shift in $\theta$ is observable which characterizes the nature of this "new" effect as explained above.

Since the topological Hall effect in these calculations is significantly larger than the anomalous Hall effect (largest for $\left.\theta=0^{\circ}, 180^{\circ}\right)$, the "new" contribution to the Hall effect in kagome magnets can be understood mainly as a topological Hall effect caused by a virtually tilted texture.

\section{G. Phase of negative vector spin chirality}

Coming back to the experiments in $\mathrm{Mn}_{3} \mathrm{Sn}$ [16] and $\mathrm{Mn}_{3} \mathrm{Ge}$ [17] where this "new" anomalous Hall effect has been measured recently, we have also investigated the phase of negative vector spin chirality, $\kappa=-1$. The results are shown in Fig. 5. In these materials the calculated Hall conductivity is smaller compared to the phase of positive chirality but it is still considerable. The general statements from above hold also in this system: For a coplanar configuration, the spin expectation value is tilted out of the plane [cf. Fig. 5(j)] which can be related to the emergence of the Hall effect [cf. Fig. 5(g)]. Also, there exists a tilted virtual texture $\left\{\boldsymbol{m}_{i}\right\}$ that determines the transport properties and a critical compensation angle $\theta_{c}$ which is smaller here compared to the $\kappa=+1$ system $\left[\theta_{c} \approx\right.$ $90.15^{\circ}$ for $\lambda=0.2 t$ as visible in Fig. 5(i)].

\section{H. Comparison to the literature and validity of the model}

Before we conclude, we want to discuss a few details that are important to realize in order to understand that our established mechanism is indeed the origin of the anomalous Hall effect in kagome magnets.

In the introduction we have mentioned several publications that report an anomalous Hall effect in kagome systems theoretically and experimentally. While these systems are mostly considered to be coplanar, actually, a small out-of-plane moment is calculated [14,30] or measured [16,17] (more than two orders of magnitude smaller than the actual magnetic moment of Mn). However, in these publications it is argued correctly that this moment is too small to explain the emergence of the calculated and measured anomalous Hall effect. In one of the first-principles calculations in Ref. [14] the system has even been artificially fixed in the perfectly coplanar configuration. As a consequence, the Hall conductivity only changed from $\sigma_{x y}=218 \Omega^{-1} \mathrm{~cm}^{-1}$ to $\sigma_{x y}=217 \Omega^{-1} \mathrm{~cm}^{-1}$ and not to zero which rules out the possibility to explain the Hall effect by means of an anomalous or topological Hall effect based on the actual magnetic texture. This means that an alternative mechanism must be present, which we have revealed in our paper.

In the study by Chen et al. [14] also a first investigation of the influence of an out-of-plane tilting of the texture on the Hall effect has been given by means of first-principles calculations. While they only calculated a few angles, it seems like the anomalous Hall effect is zero for several configurations. It would be interesting to have a more detailed curve and also the 
energy dependence to compare it to our results. The existence of several zeros could be attributed to a (random) compensation of the electron and hole carrier densities but could also stem from the existence of carriers of different orbital character. In this case, the hopping amplitude $t$ would differ between the carriers and electrons with different orbital character would experience different virtual tilting angles. This would imply that different locations of the Fermi energy (e.g., changed via doping or gating) would result in different virtual textures since the ratio of the carrier densities of electrons with different orbital characters changes. Furthermore, if the hopping amplitudes have different signs, even a compensation of the virtual tilting can occur resulting in additional zeros in the Hall conductivity. In the present model (as established in Refs. $[14,18,26,28])$ only a subspace of the total Hamiltonian is considered, which is why the virtual tilting angle is independent of the Fermi energy and only two zero transitions at $\Delta \theta_{c}$ and $\theta_{c}=90^{\circ}+\Delta \theta_{c}$ occur.

Finally, we want to address an apparent invalidity of the model considered in Refs. [14,18,26,28]. This model (that we also consider in the present paper for $\theta=90^{\circ}$ ) aims at discussing the anomalous Hall effect upon considering a coplanar magnetic texture. However, if we recall the results from the coplanar radial fixed magnetic texture (Fig. 2), we have found that while the fixed magnetic texture $\left\{\boldsymbol{m}_{i}\right\}$ exhibits no net moment, the reciprocal-space spin texture $\langle\boldsymbol{s}(\boldsymbol{k})\rangle$ of the conduction electrons does. This would mean that the total magnetic moment is finite and that a magnetization would be measured. This puts the purpose of the considered model in these publications in question: The aim of these publications was to show that an anomalous Hall effect emerges in a compensated system. However, for $\theta=90^{\circ}$, this tight-binding model does not actually consider a vanishing net magnetization (taking into account both the fixed magnetic texture and the $k$-dependent conduction electron spin texture) which is why the results of these publications do not actually serve their purpose, since one could argue that the finite Hall conductivity is not surprising even by conventional means.

However, as we show, for every tilted configuration, except for $\theta=\theta_{c}$, the Hall signal is nonzero which means that one can construct configurations for which the net moment of the fixed texture is compensated by the spin texture and still the Hall response is finite. In other words, a tweaked version of the model can sufficiently describe the completely compensated situation in which the net magnetization is absent. The fixed texture has to be considered with a small outof-plane component $\theta \neq 90^{\circ}$ but different from the critical angle $\theta \neq \theta_{c}$.

\section{CONCLUSION}

In summary, we have revealed microscopically how the anomalous Hall effect arises in kagome magnets like $\mathrm{Mn}_{3} X(\mathrm{~N})$. The Hamiltonian describes a virtual texture that determines the Hall conductivity instead of the actually measurable texture. For the coplanar radial-type arrangement, this texture is tilted out-of-plane due to the spin-orbit coupling. The virtual texture is characterized by a net moment and a net scalar spin chirality which give rise to a "virtual" anomalous and a "virtual" topological Hall effect, respectively. Our explanation is in agreement with the recent experimental findings in $\mathrm{Mn}_{3} \mathrm{Sn}$ [16] and $\mathrm{Mn}_{3} \mathrm{Ge}$ [17].

Our results go beyond conventional symmetry analyses and show precisely if and how certain symmetries are broken by the texture and the spin-orbit coupling. Especially insightful is our result for a noncoplanar system characterized by the critical azimuthal angle $\theta=\theta_{c}$ [Fig. 3(f)]. The virtual tilting due to the spin-orbit coupling leads to a coplanar virtual texture. Consequently, the Hall signal is absent for this critical configuration. While a magnetic point group analysis would tell that the Hall signal is allowed, our established microscopic mechanism allows us to understand the absence due to a preserved $T M$ symmetry of the virtual texture and the Hamiltonian of the here considered model.

As discussed, our model considers only a single orbital per lattice site, while the full system is characterized by electrons with different orbital character. In this case, due to the different hopping amplitudes [cf. Eq. (9)], different carriers cause different tilting angles of the (now orbital-dependent) virtual texture, which even allows for a compensation. This may explain why in kagome materials with a positive vector spin chirality (like $\mathrm{Mn}_{3} \mathrm{Rh}, \mathrm{Mn}_{3} \mathrm{Ir}, \mathrm{Mn}_{3} \mathrm{Pt}$ ) no such Hall effect has been measured yet, even though it is allowed by conventional symmetry analyses and was predicted more than 6 years ago. Tuning the Fermi energy by gating or doping can change the ratio of different carriers and may allow us to tune the Hall effect in these materials.

\section{ACKNOWLEDGMENTS}

This work is supported by CRC/TRR 227 of Deutsche Forschungsgemeinschaft (DFG). O.B. performed the tightbinding calculations with the help of B.G. B.G. established the transformation of the spin-orbit coupling and the virtually tilted texture. B.G. wrote the manuscript with significant inputs from all authors. O.B. prepared the figures. All authors discussed the results. B.G. and I.M. planned and supervised the project.

\section{APPENDIX: TRANSFORMATION: EQUIVALENCE OF SPIN-ORBIT COUPLING AND A TILTED MAGNETIC TEXTURE}

In the main text we have claimed that the spin-orbit coupling term behaves like a virtual tilting of the magnetic texture out of the kagome plane. In the following we derive this equivalence explicitly.

We start from the full Hamiltonian that includes hopping, Hund's coupling, and spin-orbit coupling terms. Now we apply a transformation

$$
U_{i}^{\dagger}=\left(\begin{array}{cc}
\cos \left(\frac{\alpha}{2}\right) & \sin \left(\frac{\alpha}{2}\right) e^{-i \Phi_{i}} \\
-\sin \left(\frac{\alpha}{2}\right) e^{i \Phi_{i}} & \cos \left(\frac{\alpha}{2}\right)
\end{array}\right)
$$

that is unitary,

$$
U_{i}^{\dagger} U_{i}=1
$$

This transformation reorients the coordinate system for the magnetic moments of the fixed texture at every lattice site. Instead of aligning the $z$ axis with the local moment, as has been done in Refs. [31-33], we tilt the $z$ axis (now labeled 


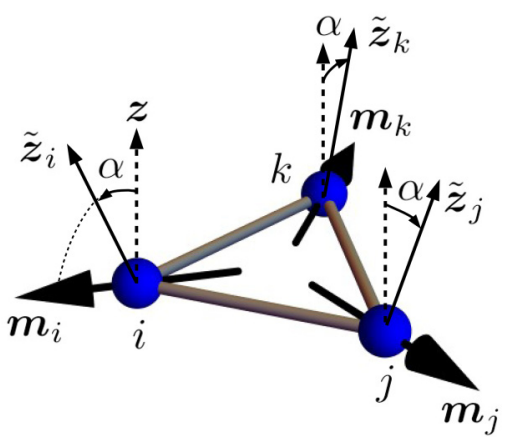

FIG. 6. Local rotation of the coordinate system as unitary transformation. The dashed coordinate system at each lattice cite $i, j, k$ is rotated about the same angle $\alpha$ in the plane spanned by $z$ and the local magnetic moment $\boldsymbol{m}_{i}, \boldsymbol{m}_{j}, \boldsymbol{m}_{k}$, respectively. The $z$ axes of the reoriented, site-dependent coordinate systems are visualized by the solid arrows $\tilde{z}_{i}, \tilde{z}_{j}, \tilde{z}_{k}$. In these coordinate systems the magnetic moments are characterized by a different azimuthal angle $\theta-\alpha$; i.e., the texture is virtually tilted.

$\tilde{z})$ by an angle $\alpha$ toward the magnetic moment, as shown in Fig. 6,

$$
\tilde{z}_{i}=\left(\cos \Phi_{i} \sin \alpha, \sin \Phi_{i} \sin \alpha, \cos \alpha\right)^{\mathrm{T}} .
$$

Here $\Phi_{i}$ is the polar angle of the fixed moment $\boldsymbol{m}_{i}$ at site $i$.

The electron operator $c_{i}$ is transformed to the new operator $d_{i}$ as

$$
c_{i}=U_{i} d_{i}
$$

First, we analyze how the Hund's coupling term transforms,

$$
\begin{gathered}
c_{i}^{\dagger}\left(\boldsymbol{m}_{i} \cdot \boldsymbol{\sigma}\right) c_{i}=d_{i}^{\dagger} U_{i}^{\dagger}\left(\boldsymbol{m}_{i} \cdot \boldsymbol{\sigma}\right) U_{i} d_{i}, \\
U_{i}^{\dagger}\left(\boldsymbol{m}_{i} \cdot \boldsymbol{\sigma}\right) U_{i}=\left(\begin{array}{cc}
\cos (\theta-\alpha) & \sin (\theta-\alpha) e^{-i \Phi_{i}} \\
\sin (\theta-\alpha) e^{i \Phi_{i}} & -\cos (\theta-\alpha)
\end{array}\right) .
\end{gathered}
$$

As expected by geometric considerations, the azimuthal angle of the magnetic moment in this new coordinate system is decreased by $\alpha$ and the polar angle remains the same. Due to the application of the transformation, the spin is measured differently; the magnetic texture effectively acts as a new texture $\left\{\tilde{\boldsymbol{m}}_{i}\right\}$ :

$$
\begin{gathered}
c_{i}^{\dagger}\left(\boldsymbol{m}_{i} \cdot \boldsymbol{\sigma}\right) c_{i}=d_{i}^{\dagger}\left(\tilde{\boldsymbol{m}}_{i} \cdot \boldsymbol{\sigma}\right) d_{i}, \\
\tilde{\boldsymbol{m}}_{i}=\left(\begin{array}{c}
\cos \left(\Phi_{i}\right) \sin (\theta-\alpha) \\
\sin \left(\Phi_{i}\right) \sin (\theta-\alpha) \\
\cos (\theta-\alpha)
\end{array}\right) .
\end{gathered}
$$

Of course, the unitary transformation has to be applied also to the other two terms to leave the physics of the system unchanged. Therefore, the effect of this new, tilted texture has to be compensated by the other terms in the Hamiltonian. Next, we transform the hopping term:

$$
\begin{gathered}
t c_{i}^{\dagger} c_{j}=t d_{i}^{\dagger} U_{i}^{\dagger} U_{j} d_{j}=t U_{i}^{\dagger} U_{j} d_{i}^{\dagger} d_{j}, \\
U_{i}^{\dagger} U_{j}=\left(\begin{array}{cc}
\cos ^{2}\left(\frac{\alpha}{2}\right)+\sin ^{2}\left(\frac{\alpha}{2}\right) e^{-i\left(\Phi_{i}-\Phi_{j}\right)} & \frac{1}{2} \sin (\alpha)\left(e^{-i \Phi_{i}}-e^{-i \Phi_{j}}\right) \\
-\frac{1}{2} \sin (\alpha)\left(e^{i \Phi_{i}}-e^{i \Phi_{j}}\right) & \cos ^{2}\left(\frac{\alpha}{2}\right)+\sin ^{2}\left(\frac{\alpha}{2}\right) e^{i\left(\Phi_{i}-\Phi_{j}\right)}
\end{array}\right) .
\end{gathered}
$$

The diagonal terms describe a hopping with a scaled hopping amplitude $t_{i j}^{\text {eff }}$. This hopping has a complex phase which is opposite for spin-up and spin-down (in this basis) electrons. For a small $\alpha$, the effective hopping converges to $t$.

The off-diagonal terms have the shape of the spin-orbit coupling. For the radial texture they can be simplified to

$$
-i \frac{\sqrt{3}}{2} \sin (\alpha) \boldsymbol{n}_{i j} \cdot \boldsymbol{\sigma}
$$

Lastly, we transform the original spin-orbit coupling term. Again, the result is a matrix with effective hooping terms on the diagonal and effective spin-orbit coupling terms on the off-diagonal:

$$
\begin{gathered}
c_{i}^{\dagger}\left(\vec{n}_{i j} \cdot \vec{\sigma}\right) c_{j}=d_{i}^{\dagger} U_{i}^{\dagger}\left(\vec{n}_{i j} \cdot \vec{\sigma}\right) U_{j} d_{j} \\
U_{i}^{\dagger}\left(\boldsymbol{n}_{i j} \cdot \boldsymbol{\sigma}\right) U_{j}= \\
\cos (\alpha) \boldsymbol{n}_{i j} \cdot \boldsymbol{\sigma}+\sin (\alpha) \\
\times\left(\begin{array}{cc}
e^{-i \frac{1}{2}\left(\Phi_{i}-\Phi_{j}\right)} & 0 \\
0 & -e^{i \frac{1}{2}\left(\Phi_{i}-\Phi_{j}\right)}
\end{array}\right) .
\end{gathered}
$$

Summarizing the transformation, the Hamiltonian

$$
\begin{aligned}
H= & t \sum_{\langle i, j\rangle} c_{i}^{\dagger} c_{j}+m \sum_{i} c_{i}^{\dagger}\left(\boldsymbol{m}_{i} \cdot \boldsymbol{\sigma}\right) c_{i} \\
& +i \lambda \sum_{\langle i, j\rangle} c_{i}^{\dagger}\left(\boldsymbol{n}_{i j} \cdot \boldsymbol{\sigma}\right) c_{j}
\end{aligned}
$$

has been transformed to

$$
\begin{aligned}
H= & \sum_{\langle i, j\rangle} t_{i j}^{\mathrm{eff}} d_{i}^{\dagger} d_{j}+m \sum_{i} d_{i}^{\dagger}\left(\tilde{\boldsymbol{m}}_{i} \cdot \boldsymbol{\sigma}\right) d_{i} \\
& +i \tilde{\lambda} \sum_{\langle i, j\rangle} d_{i}^{\dagger}\left(\boldsymbol{n}_{i j} \cdot \boldsymbol{\sigma}\right) d_{j},
\end{aligned}
$$

with

$$
\begin{aligned}
t_{i j}^{\mathrm{eff} \uparrow}= & t\left[\cos ^{2}\left(\frac{\alpha}{2}\right)+\sin ^{2}\left(\frac{\alpha}{2}\right) e^{\mp i\left(\Phi_{i}-\Phi_{j}\right)}\right] \\
& \pm i \lambda e^{\mp i \frac{1}{2}\left(\Phi_{i}-\Phi_{j}\right)} \sin (\alpha), \\
\tilde{\lambda}= & -\frac{\sqrt{3}}{2} \sin (\alpha) t+\cos (\alpha) \lambda,
\end{aligned}
$$




$$
\tilde{\boldsymbol{m}}_{i}=\left(\begin{array}{c}
\cos \left(\Phi_{i}\right) \sin (\theta-\alpha) \\
\sin \left(\Phi_{i}\right) \sin (\theta-\alpha) \\
\cos (\theta-\alpha)
\end{array}\right)
$$

This means that a tilting of the texture is equivalent to the emergence of an effective spin-orbit coupling and a modified hopping amplitude, and vice versa. At a critical tilting angle $\alpha_{c}$ the spin-orbit coupling is compensated,

$$
0 \stackrel{!}{=} \tilde{\lambda}=-\frac{\sqrt{3}}{2} \sin \left(\alpha_{c}\right) t+\cos \left(\alpha_{c}\right) \lambda
$$

This means that gauging away the spin-orbit coupling is equivalent to tilting the virtual texture by $-\alpha_{c}$. Therefore, a critical angle $\Delta \theta_{c}=+\alpha_{c}$ of the actual texture compensates this tilt and leads to a coplanar virtual texture that does not exhibit a Hall effect,

$$
\Delta \theta_{c}=\alpha_{c}=\arctan \frac{\lambda}{t \sqrt{3} / 2} .
$$

For a general $\kappa=+1$ configuration, the angle is given by

$$
\Delta \theta_{c}=\alpha_{c}=\arctan \left[\frac{\lambda}{t \sqrt{3} / 2} \cos (\Delta \Phi)\right] .
$$

[1] E. H. Hall, On a new action of the magnet on electric currents, Am. J. Math. 2, 287 (1879).

[2] E. H. Hall, XVIII. On the "rotational coefficient" in nickel and cobalt, The London, Edinburgh, and Dublin Philosophical Magazine and Journal of Science 12, 157 (1881).

[3] N. Nagaosa, J. Sinova, S. Onoda, A. H. MacDonald, and N. P. Ong, Anomalous Hall effect, Rev. Mod. Phys. 82, 1539 (2010).

[4] J. Smit, The spontaneous Hall effect in ferromagnetics I, Physica 21, 877 (1955).

[5] J. Smit, The spontaneous Hall effect in ferromagnetics II, Physica 24, 39 (1958).

[6] L. Berger, Side-jump mechanism for the Hall effect of ferromagnets, Phys. Rev. B 2, 4559 (1970).

[7] R. Karplus and J. M. Luttinger, Hall effect in ferromagnetics, Phys. Rev. 95, 1154 (1954).

[8] M. V. Berry, Quantal phase factors accompanying adiabatic changes, Proc. R. Soc. London A 392, 45 (1984).

[9] J. Zak, Berry's Phase for Energy Bands in Solids, Phys. Rev. Lett. 62, 2747 (1989).

[10] P. Bruno, V. K. Dugaev, and M. Taillefumier, Topological Hall Effect and Berry Phase in Magnetic Nanostructures, Phys. Rev. Lett. 93, 096806 (2004).

[11] S. Mühlbauer, B. Binz, F. Jonietz, C. Pfleiderer, A. Rosch, A. Neubauer, R. Georgii, and P. Böni, Skyrmion lattice in a chiral magnet, Science 323, 915 (2009).

[12] A. Neubauer, C. Pfleiderer, B. Binz, A. Rosch, R. Ritz, P. G. Niklowitz, and P. Böni, Topological Hall Effect in the A Phase of MnSi, Phys. Rev. Lett. 102, 186602 (2009).

[13] N. Nagaosa and Y. Tokura, Topological properties and dynamics of magnetic skyrmions, Nat. Nanotechnol. 8, 899 (2013).

[14] H. Chen, Q. Niu, and A. H. MacDonald, Anomalous Hall Effect Arising from Noncollinear Antiferromagnetism, Phys. Rev. Lett. 112, 017205 (2014).

[15] J. Kübler and C. Felser, Non-collinear antiferromagnets and the anomalous Hall effect, Europhys. Lett. 108, 67001 (2014).

[16] S. Nakatsuji, N. Kiyohara, and T. Higo, Large anomalous Hall effect in a non-collinear antiferromagnet at room temperature, Nature (London) 527, 212 (2015).

[17] A. K. Nayak, J. E. Fischer, Y. Sun, B. Yan, J. Karel, A. C. Komarek, C. Shekhar, N. Kumar, W. Schnelle, J. Kübler et al., Large anomalous Hall effect driven by a nonvanishing Berry curvature in the noncolinear antiferromagnet $\mathrm{Mn}_{3} \mathrm{Ge}$, Sci. Adv. 2, e1501870 (2016)
[18] Y. Zhang, Y. Sun, H. Yang, J. Železný, S. P. P. Parkin, C. Felser, and B. Yan, Strong anisotropic anomalous Hall effect and spin Hall effect in the chiral antiferromagnetic compounds $\mathrm{Mn}_{3} X$ ( $X=\mathrm{Ge}, \mathrm{Sn}, \mathrm{Ga}, \mathrm{Ir}, \mathrm{Rh}$, and Pt), Phys. Rev. B 95, 075128 (2017).

[19] M. Seemann, D. Ködderitzsch, S. Wimmer, and H. Ebert, Symmetry-imposed shape of linear response tensors, Phys. Rev. B 92, 155138 (2015).

[20] Note that experiments $[16,17]$ as well as first-principles calculations $[14,30]$ find a very small net moment on the order of $10^{-2} \mu_{B}$ per unit cell for these systems. As stated in these publications, this moment is too small to explain the emergence of the anomalous Hall effect by conventional means. In Ref. [14] it has even been shown that the effect is only slightly reduced from $\sigma_{x y}=218 \Omega^{-1} \mathrm{~cm}^{-1}$ to $\sigma_{x y}=217 \Omega^{-1} \mathrm{~cm}^{-1}$ when the magnetic texture is artificially fixed in the plane in first-principles calculations, so that the moment is completely compensated. Therefore, an unconventional mechanism must be present.

[21] C. L. Kane and E. J. Mele, Quantum Spin Hall Effect in Graphene, Phys. Rev. Lett. 95, 226801 (2005).

[22] E. F. Bertaut, D. Fruchart, J. P. Bouchaud, and R. Fruchart, Diffraction neutronique de $\mathrm{Mn}_{3} \mathrm{GaN}$, Solid State Commun. 6, 251 (1968).

[23] D. Fruchart and E. F. Bertaut, Magnetic studies of the metallic perovskite-type compounds of manganese, J. Phys. Soc. Jpn. 44, 781 (1978).

[24] W. Feng, G.-Y. Guo, J. Zhou, Y. Yao, and Q. Niu, Large magneto-optical Kerr effect in noncollinear antiferromagnets $\mathrm{Mn}_{3} X(X=\mathrm{Rh}, \mathrm{Ir}, \mathrm{Pt})$, Phys. Rev. B 92, 144426 (2015).

[25] G.-Y. Guo and T.-C. Wang, Large anomalous Nernst and spin Nernst effects in the noncollinear antiferromagnets $\operatorname{Mn}_{3} X(X=$ Sn, Ge, Ga), Phys. Rev. B 96, 224415 (2017).

[26] X. Zhou, J.-P. Hanke, W. Feng, F. Li, G.-Y. Guo, Y. Yao, S Blügel, and Y. Mokrousov, Spin-order dependent anomalous Hall effect and magneto-optical effect in the noncollinear antiferromagnets $\mathrm{Mn}_{3} X \mathrm{~N}$ with $X=\mathrm{Ga}, \mathrm{Zn}, \mathrm{Ag}$, or Ni, Phys. Rev. B 99, 104428 (2019).

[27] See Supplemental Material at http://link.aps.org/supplemental/ 10.1103/PhysRevResearch.2.033112 for five supplementary figures. In Fig. S1 we present the band structure and the density of states of the structural kagome lattice. In Fig. S2 we compare the k-resolved spin expectation values for various configurations, and in Fig. S3 we show that the system with the 
toroidal texture is not affected by the spin-orbit coupling and does not exhibit an anomalous Hall effect. Furthermore, in Fig. S4 we analyze the tilted radial texture for a constant occupation number, and in Fig. S5 we confirm Eq. (9) numerically.

[28] Y. Zhang, J. Železný, Y. Sun, J. van den Brink, and B. Yan, Spin Hall effect emerging from a noncollinear magnetic lattice without spin-orbit coupling, New J. Phys. 20, 073028 (2018).

[29] D. J. Thouless, M. Kohmoto, M. P. Nightingale, and M. Den Nijs, Quantized Hall Conductance in a Two-Dimensional Periodic Potential, Phys. Rev. Lett. 49, 405 (1982).

[30] D. Zhang, B. Yan, S.-C. Wu, J. Kübler, G. Kreiner, S. S. P. Parkin, and C. Felser, First-principles study of the structural stability of cubic, tetragonal and hexagonal phases in $\mathrm{Mn}_{3} Z$ ( $Z=\mathrm{Ga}, \mathrm{Sn}$ and $\mathrm{Ge}$ ) Heusler compounds, J. Phys.: Condens. Matter 25, 206006 (2013).

[31] K. Hamamoto, M. Ezawa, and N. Nagaosa, Quantized topological Hall effect in skyrmion crystal, Phys. Rev. B 92, 115417 (2015).

[32] B. Göbel, A. Mook, J. Henk, and I. Mertig, Unconventional topological Hall effect in skyrmion crystals caused by the topology of the lattice, Phys. Rev. B 95, 094413 (2017).

[33] B. Göbel, A. Mook, J. Henk, and I. Mertig, The family of topological Hall effects for electrons in skyrmion crystals, Eur. Phys. J. B 91, 179 (2018). 